\title{
O Efeito Anti-Hipertensivo de Sauromatum Guttatum Mediado por Efeitos Vasorrelaxante e Depressivos Miocárdicos
}

\author{
Antihypertensive Activity of Sauromatum guttatum Mediated by Vasorelaxation and Myocardial \\ Depressant Effects \\ Rabia Bibi, ${ }^{1}$ Umme Salma, ${ }^{1,2}$ Kashif Bashir, ${ }^{1}$ Taous Khan, ${ }^{1}$ Abdul Jabbar Shah ${ }^{1}$ (10 \\ COMSATS University Islamabad - Abbottabad Campus - Department of Pharmacy, ${ }^{1}$ Abbottabad, Khyber Pakhtunkhwa - Paquistão \\ Ibadat International University Islamabad, ${ }^{2}$ Islamabad - Paquistão
}

\section{Resumo}

Fundamento: A Sauromatum guttatum (S. guttatum) é utilizado no tratamento de doenças do sangue e supostamente tem atividade espasmolítica através da inibição dos canais de $\mathrm{Ca}^{2+}$.

Objetivos: O objetivo deste estudo foi investigar o potencial anti-hipertensivo de S. guttatum em modelo de rato SpragueDawley (SD) com hipertensão induzida por dieta com alto teor de sal (HIDATS).

Métodos: Ratos SD foram divididos em normotensos, hipertensos e grupos tratados com verapamil e $S$. guttatum. Extrato bruto de $S$. guttatum (Sg.B) (100, 150 e $300 \mathrm{mg} / \mathrm{kg} / \mathrm{dia})$ e verapamil $(5,10$ e $15 \mathrm{mg} / \mathrm{kg} / \mathrm{dia})$ foram administrados por via oral junto com $\mathrm{NaCl}$. Anéis aórticos e faixas do átrio direito de ratos normotensos foram utilizados para investigar os mecanismos subjacentes. O nível de significância estatística adotado foi de $5 \%$.

Resultados: A pressão arterial média diminuiu nos grupos hipertensos tratados com Sg.B e verapamil de forma dose-dependente $(\mathrm{p}<0,001)$. No estudo de reatividade vascular, a acetilcolina induziu relaxamentos com valor $\mathrm{CE}_{50}$ de $0,6 \mu \mathrm{g} / \mathrm{mL}(0,3-1,0) \mathrm{em}$ ratos hipertensos tratados com Sg.B $(300 \mathrm{mg} / \mathrm{kg})$, sugerindo preservação endotelial. Em aorta isolada de rato normotenso, 0 Sg.B exibiu vasorrelaxamento com valor de $\mathrm{CE}_{50}$ de $0,15 \mathrm{mg} / \mathrm{mL}(0,10-0,20)$, após ablação por desnudamento endotelial ou pré-tratamento com L-NAME e atropina. $\mathrm{O}$ tratamento com Sg.B causou relaxamento contra contraçóes induzidas por $\mathrm{K}^{+}$alto, como o verapamil. O Sg.B mostrou efeitos inotrópicos (82\%) e cronotrópicos (56\%) negativos em preparações isoladas atriais de ratos reduzidas com atropina. A avaliação fitoquímica indicou a presença de alcaloides, flavonoides e taninos.

Conclusão: O S. guttatum possui efeito vasodilatador através da preservação da função endotelial, liberação de NO mediada pelo receptor muscarínico e inibição do movimento de $\mathrm{Ca}^{2+}$, enquanto o efeito depressor do miocárdio atrial pode estar ligado ao receptor muscarínico. Esses achados fornecem a base farmacológica para o uso do extrato de $S$. guttatum como um medicamento anti-hipertensivo.

Palavras-chave: Ratos; Anti-Hipertensivos; Sauromatum guttatum; Pressão Arterial; Hipertensão; Vasodilatação; Bloqueio do canal de $\mathrm{Ca}^{2+}$; Desempenho cardíaco.

\footnotetext{
Abstract

Background: Sauromatum guttatum (S. guttatum) is used in the treatment of blood disorders and reportedly has a spasmolytic activity through $\mathrm{Ca}^{2+}$ channel inhibition.

Objectives: The aim of this study was to investigate the antihypertensive potential of S. guttatum in high salt-induced hypertensive SpragueDawley (SD) rat model (HSHRs)

Methods: SD rats were divided into normotensive, hypertensive, S. guttatum and verapamil treated groups. S. guttatum crude extract (Sg.Cr) (100, 150 and $300 \mathrm{mg} / \mathrm{kg} /$ day) and verapamil (5, 10 and $15 \mathrm{mg} / \mathrm{kg} /$ day) were administered orally along with NaCl. Aortic rings and right atrial strips from normotensive rats were used to investigate the underlying mechanisms. The level of statistical significance was set at 5\%.

Results: Mean arterial pressure decreased in the Sg.Cr and verapamil-treated hypertensive groups in a dose-dependent manner ( $p<0.001)$. In the vascular reactivity study, acetylcholine induced relaxations with an $E C_{50}$ value of $0.6 \mu \mathrm{g} / \mathrm{mL}(0.3-1.0)$ in Sg.Cr-treated hypertensive rats $\left(300 \mathrm{mg} / \mathrm{kg}\right.$ ), suggesting endothelial preservation. In isolated normotensive rat aorta, Sg.Cr-treated rats showed vasorelaxation with an $\mathrm{EC}_{50}$ value of $0.15 \mathrm{mg} / \mathrm{mL}(0.10-0.20)$, ablated by endothelial denudation or pretreatment with -NAME and atropine. Sg.Cr treatment caused relaxation against high $\mathrm{K}^{+}$-induced contractions, like verapamil. Sg.Cr showed negative inotropic (82\%) and chronotropic effects (56\%) in isolated rat atrial preparations reduced with atropine. The phytochemical investigation indicated presence of alkaloids, flavonoids and tannins.
}

Correspondência: Abdul Jabbar Shah •

COMSATS University Islamabad - Abbottabad Campus - Department of Pharmacy, Abbottabad, Khyber Pakhtunkhwa - Paquistão

E-mail: jabbarshah@cuiatd.edu.pk

Artigo recebido em 29/01/2020, revisado em 28/11/2020, aceito em 27/01/2021

DOI: https://doi.org/10.36660/abc.20200055 
Conclusion: S. guttatum has a vasodilatory effect through endothelial function preservation, muscarinic receptor-mediated $\mathrm{NO}$ release and Ca ${ }^{2+}$ movement inhibition, while atrial myocardial depressant effect can be linked to the muscarinic receptor. These findings provide pharmacological base for using S. guttatum extract as an antihypertensive medication.

Keywords: Rats; Antihypertensive; Sauromatum guttatum; Blood Pressure; Hypertension; Vasodilatation; Calcium Channel Blockers; Cardiac Performance.

Full texts in English - http://www.arquivosonline.com.br

\section{Introdução}

A hipertensão é um fator de risco importante para doenças cardiovasculares e mortalidade devido aos danos em órgãosalvo. ${ }^{1}$ Existem muitos fatores ambientais que contribuem para a etiologia da hipertensão, incluindo ingestão elevada de sal. A alta ingestão de sal continua sendo o fator mais importante na etiologia da hipertensão em humanos. ${ }^{2}$ Em ratos, a alta ingestão de sal também promove a hipertensão, fornecendo um modelo conveniente para estudar a hipertensão humana. ${ }^{3} \mathrm{O}$ consumo contínuo de uma dieta rica em sal leva à disfunção endotelial, o que pode representar um fator de risco particularmente significativo no desenvolvimento da hipertensão, ${ }^{4}$ afetando negativamente a qualidade de vida. ${ }^{5}$ As medidas para o manejo da hipertensão incluem ajuste no estilo de vida, modificação da dieta, exercícios físicos, bem como terapias convencionais e alternativas, incluindo remédios fitoterápicos. ${ }^{6-8}$ A Sauromatum guttatum (S. guttatum) pertence à família Araceae, e é comumente conhecida como "lírio vodu" e "monarca do Oriente". A Sauromatum guttatum é conhecida como "Sanp ki Booti" no Paquistão e na Índia, onde é onipresente. A S. guttatum é tradicionalmente utilizada para tratar inflamação, dificuldades respiratórias, ${ }^{9}$ problemas gástricos, ${ }^{10}$ tuberculose, doenças do sangue, picadas de cobra e infecções de pele. ${ }^{11}$ A $S$. guttatum contém lectinas, dimetilsulfetos, cariofileno, indol, escatol, amônia, trimetilamina e aminas primárias. ${ }^{12-14}$ Os cormos ou bulbos contêm carbono, magnésio, enxofre, oxigênio, fósforo, potássio e cloro. Estudos in vitro revelaram atividades mitogênica, ${ }^{15}$ antiproliferativa, ${ }^{16}$ herbicida, ${ }^{17}$ inibidora da lipoxigenase, ${ }^{18}$ antioxidante, antibacteriana, ${ }^{19}$ espasmolítica ${ }^{18,20}$ e inseticida da $S$. guttatum. ${ }^{17,21}$ É tradicionalmente utilizada para o tratamento de doenças do sangue. Foi relatado anteriormente que seu efeito espasmolítico é mediado pelo bloqueio de entrada de $\mathrm{Ca}^{2+}$ na musculatura lisa do intestino. ${ }^{20}$ Os bloqueadores de entrada de $\mathrm{Ca}^{2+}$ também têm papel terapêutico importante no tratamento da hipertensão. Todas essas observações fornecem uma base sólida para nossa hipótese de que o extrato de $S$. guttatum pode ter propriedades anti-hipertensivas. O objetivo deste estudo foi investigar o potencial anti-hipertensivo da S. guttatum e revelar os mecanismos subjacentes utilizando métodos in vivo e in vitro.

\section{Materiais e métodos}

\section{Preparação do extrato bruto e análise fitoquímica}

Cormos de $S$. guttatum foram adquiridos em Nathia Gali, Paquistão (junho-julho de 2018), identificados e validados pelo Dr. Abdul Nazir, Professor Assistente do Departamento de
Biotecnologia, COMSATS University Islamabad, Abbottabad Campus, Paquistão. CHUA-112 é o código de voucher para o espécime no herbário do Departamento de Farmácia, COMSATS University Islamabad, Abbottabad Campus, Paquistão. Cormos frescos foram picados e submetidos à secagem na sombra em temperatura ambiente. Em seguida, o material seco foi pulverizado, mergulhado em metanol aquoso (70\%) com agitação ocasional durante quinze, sete e três dias. O macerado foi filtrado em um tecido de musselina e, em seguida, com um papel filtro qualitativo (Whatman, Grau 1). ${ }^{22}$ Esse processo foi repetido três vezes. Em seguida, um evaporador rotativo $\left(-760 \mathrm{mmHg}\right.$ a $\left.37^{\circ} \mathrm{C}\right)$ foi utilizado para concentrar o extrato líquido. $\mathrm{O}$ extrato bruto foi analisado fitoquimicamente para todos os constituintes importantes, como flavonoides, alcaloides, saponinas, fenóis e taninos. ${ }^{23}$

\section{Animais}

Todos os experimentos foram realizados em conformidade com as orientações da Commission on Life Sciences, Institute of Laboratory Animal Resources, National Research Council ${ }^{24}$ e aprovadas pelo Comitê de Ética. Os ratos SpragueDawley (SD) foram mantidos no Biotério com comida e água disponíveis ad libitum.

\section{Investigações farmacológicas}

\section{Medicamentos e padrões}

Os medicamentos e padrões foram adquiridos das seguintes fontes: cloreto de acetilcolina, cloridrato de fenilefrina, sulfato de atropina e o pentotal sódico dos Laboratórios Abbott, Paquistão; o cloridrato de isoprenalina, cloreto de potássio, cloridrato de éster metílico de $\mathrm{N} \omega$ Nitro-L-arginina (L-NAME) e cloridrato de verapamil da empresa Sigma Chemicals, EUA.

\section{Estudos in vivo}

Modelo e grupos de ratos com hipertensão induzida por dieta com alto teor de sal (HIDATS)

Ratos SD (200-250 g) ( $\mathrm{n}=60$ ) foram divididos aleatoriamente em oito grupos ( $n=5-7$ em cada grupo). A amostragem foi feita por conveniência. O Grupo 1 (grupo controle normal) recebeu dieta normal. O Grupo 2 (grupo de hipertensos) recebeu $\mathrm{NaCl}(8 \%$ na dieta $+1 \%$ na água de beber) por 8 semanas. Os grupos 3-5 (grupo tratado com $\mathrm{S}$. guttatum) receberam $\mathrm{NaCl}(8 \%$ na dieta $+1 \%$ na água potável) e diferentes doses orais de extrato bruto de $S$. guttatum (100 mg/kg/dia, $150 \mathrm{mg} / \mathrm{kg} /$ dia e $300 \mathrm{mg} / \mathrm{kg} / \mathrm{dia}$ ) 
uma vez por dia durante 8 semanas. Os grupos 6 a 8 (grupo com tratamento padrão) receberam por via oral doses diárias de verapamil (5 mg/kg/dia, $10 \mathrm{mg} / \mathrm{kg}$ e $15 \mathrm{mg} / \mathrm{kg} /$ dia) junto com dieta de $\mathrm{NaCl}$ contendo $8 \% \mathrm{NaCl}+1 \% \mathrm{NaCl}$ na água de beber por 8 semanas. ${ }^{25-27}$

\section{Registro invasivo de pressão arterial em ratos com HIDATS}

A intubação traqueal de ratos SD anestesiados (pentotal, 40-100 mg/kg, IP) foi realizada com tubo de polietileno (PE20). Para monitorar a pressão arterial, a artéria carótida direita foi canulada com tubo de polietileno (PE-50) e afixada no PowerLab Data Acquisition System (ADInstrument, Austrália), através de um transdutor de pressão (MLT 0699). Uma lâmpada suspensa foi utilizada para manter a temperatura corporal do animal. A pressão arterial média foi monitorada por 30 minutos em cada grupo. ${ }^{25,28}$

\section{Perfil de peso corporal}

O peso corporal de todos os grupos foi determinado no início do experimento e posteriormente monitorado semanalmente. Após 8 semanas de tratamento, a mudança no peso corporal foi calculada.

\section{Estudos in vitro}

\section{Estudos de reatividade vascular}

Para investigar o efeito da preservação do endotélio induzido pelo extrato bruto em ratos com HIDATS, isolamos a aorta dos grupos normotenso, hipertenso e tratados. Os anéis aórticos foram suspensos em banhos teciduais (10 $\mathrm{mL})$, contendo carbogênio $\left(5 \% \mathrm{CO}_{2}\right.$ e $\left.95 \% \mathrm{O}_{2}\right)$ solução de Krebs normal aerada, composta por $\mathrm{NaCl}, 118,2 \mathrm{mM} ; \mathrm{KCl}$, 4,7 mM; $\mathrm{MgSO}_{4}, 1,2 \mathrm{mM}$; $\mathrm{KH}_{2} \mathrm{PO}_{4}, 1,3 \mathrm{mM}$; $\mathrm{NaHCO}_{3}, 25,0$ $\mathrm{mM}$; Glicose, 11,7 mM; $\mathrm{CaCl}_{2,}$ 2,5 mM, mantido a 37으. A força foi monitorada pelo PowerLab Data Acquisition System (ADInstrument, Austrália) e um amplificador em ponte (N12128) através de um transdutor de deslocamento de força (MLT 0201). Os anéis aórticos foram estabilizados em tensão isométrica a 2 g por 60-90 minutos, com troca da solução de Krebs a cada 15 minutos. Para determinar a integridade do endotélio, diferentes concentrações de acetilcolina foram utilizadas em anéis aórticos pré-constritos com fenilefrina (1 $\mu \mathrm{M})^{25,28}$

\section{Preparações isoladas de aorta de rato SD}

Os anéis aórticos foram suspensos em banhos teciduais contendo $10 \mathrm{~mL}$ de carbogênio ( $5 \% \mathrm{CO}_{2}$ e $95 \% \mathrm{O}_{2}$ ) e solução de Krebs normal aerada mantida a $37^{\circ} \mathrm{C}$, fixada no PowerLab Data Acquisition System (ADInstrument, Austrália) e um amplificador em ponte (N12128) através de um transdutor de deslocamento de força (MLT 0201). Os anéis foram equilibrados por 60-90 minutos a uma tensão isométrica de $2 \mathrm{~g}$, enquanto a solução foi trocada a cada 15 minutos. Diferentes concentrações $(0,1-10 \mathrm{mg} / \mathrm{mL})$ de $S$. guttatum foram adicionadas aos anéis pré-constritos com fenilefrina (FE).
Para determinar o mecanismo subjacente, os anéis aórticos foram pré-tratados com atropina $1 \mu \mathrm{M}$ ou L-NAME $10 \mu \mathrm{M}$ por 30 minutos. Em alguns experimentos, anéis desnudados de endotélio de ratos normotensos foram utilizados. ${ }^{25,28,29}$

\section{Preparações isoladas do átrio direito}

Os átrios direitos de ratos SD normotensos foram dissecados. As preparações atriais foram suspensas em banhos teciduais contendo $10 \mathrm{~mL}$ de solução aerada de Krebs, mantida a $32^{\circ} \mathrm{C}$, ligada ao PowerLab (ML 846) Data Acquisition System (ADInstrument, Austrália) e amplificador em ponte (N12128), através de um transdutor de força (MLT 0201). Os tecidos foram estabilizados em uma tensão de repouso de $1 \mathrm{~g}$ por 30 minutos. O envolvimento do receptor muscarínico foi estudado em preparações atriais pré-tratadas com atropina $(1 \mu \mathrm{M}){ }^{25,28}$

\section{Análise estatística}

Os dados tinham distribuição normal, como determinado pelo teste de normalidade de Shapiro-Wilk. Os dados foram expressos como média \pm desvio padrão (DP) e as medianas das concentrações efetivas (valores de $\mathrm{CE}_{50}$ ) com intervalo de confiança (IC) de 95\%. A porcentagem de mudança nos perfis de pressão arterial média (PAM) ou peso corporal foram calculados por análise de variância (ANOVA) de uma via (seguida por teste post-hoc de Tukey HSD). A porcentagem de vasorrelaxamento em ratos normotensos e hipertensos foi calculada por ANOVA de duas vias (seguida pelo teste post-hoc de Bonferroni) utilizando o software SPSS 21 (EUA). O nível de significância estatística aceito foi de 5\%.

\section{Resultados}

\section{Constituintes fitoquímicos}

A análise fitoquímica preliminar realizada no extrato de cormos de $S$. guttatum identificou alcaloides, flavonoides, fenóis, fitosteróis, saponinas e taninos.

\section{Investigação farmacológica}

\section{Estudos in vivo}

\section{Monitoramento invasivo de pressão arterial}

Os valores da pressão arterial média (PAM) medidos nos diferentes grupos experimentais são mostrados nas Figuras 1 e 2. A PAM do grupo de ratos com HIDATS apresentou elevação de $67,7 \%$ da pressão arterial em comparação ao grupo controle normotenso. Esta elevação na PAM foi revertida pelo tratamento com Sg.B de maneira dose-dependente ( $p<0,01$ ) até a concentração de $300 \mathrm{mg} / \mathrm{kg}$, onde atingiu seu efeito máximo de redução da PAM $(p<0,001)$. A PAM em ratos tratados com verapamil (5 mg/kg e $10 \mathrm{mg} / \mathrm{kg}$ ) também diminuiu de maneira dose-dependente $(p<0,01$ e $p<0,001$ respectivamente) atingindo o efeito máximo na concentração de $15 \mathrm{mg} / \mathrm{kg}(\mathrm{p}<0,001)$. 


\section{Artigo Original}

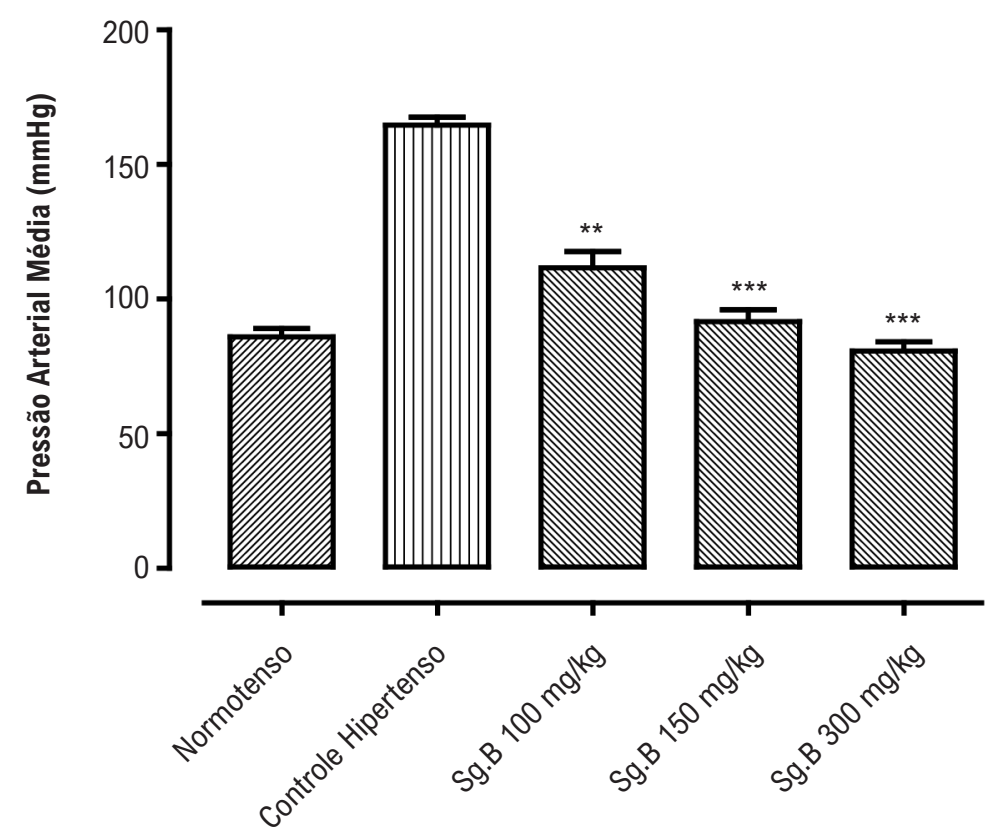

Figura 1 - Pressão arterial média em ratos normotensos, hipertensos e ratos tratados com extrato bruto de Sauromatum guttatum (Sg.Cr) para hipertensão induzida por dieta com alto tear de sal, em doses de $100 \mathrm{mg} / \mathrm{kg}, 150 \mathrm{mg} / \mathrm{kg}$ e $300 \mathrm{mg} / \mathrm{kg}$ ( $\mathrm{n}=5$ - 7; média \pm EPM). Comparado com os valores do grupo controle hipertensivo, ${ }^{* *} p<0,01 e^{* * *} p<0,001$

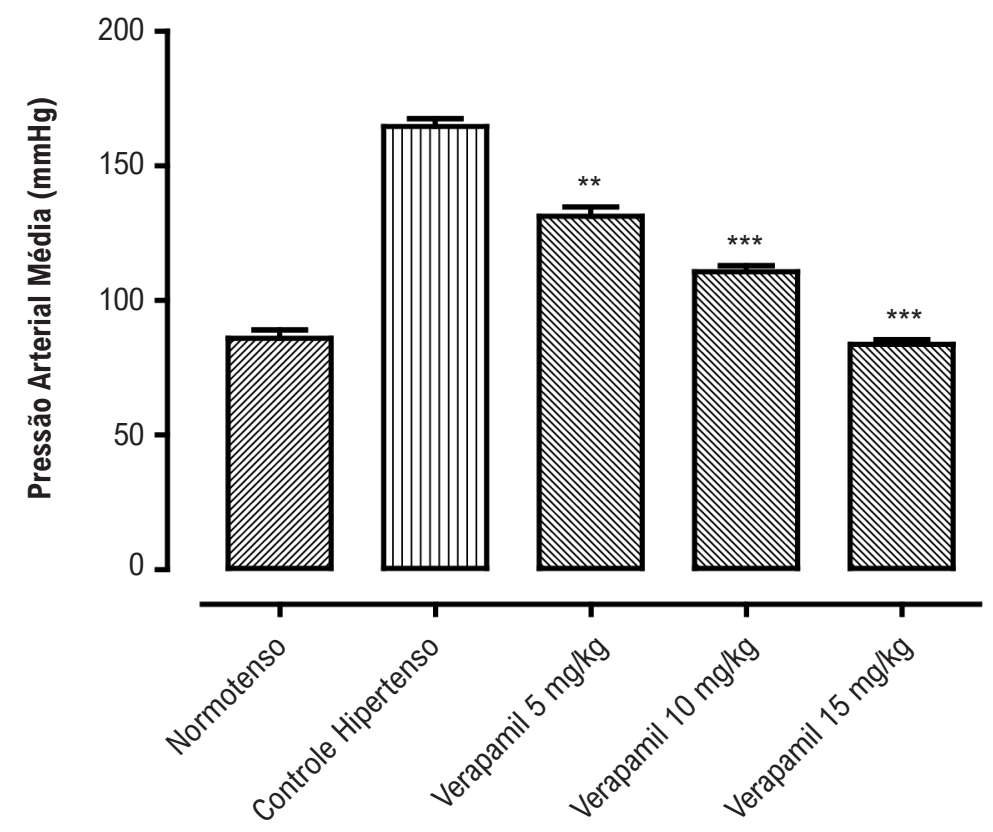

Figura 2 - Pressão arterial média em ratos normotensos, hipertensos e com hipertensão induzida por dieta com alto teor de sal tratados com verapamil nas doses de $5 \mathrm{mg} / \mathrm{kg}, 10 \mathrm{mg} / \mathrm{kg}$ e $15 \mathrm{mg} / \mathrm{kg}$ ) ( $n=5-7$; média \pm EPM). Comparado com os valores do grupo controle hipertenso, ${ }^{* *} p<0,01 e^{* * *} p<0,001$ 


\section{Perfil de peso corporal}

A alta ingestão de sal por 8 semanas causou uma diminuição significativa $(p<0,001)$ no peso corporal no grupo controle hipertenso (Tabela 1). O tratamento de ratos hipertensos com extrato bruto de Sauromatum guttatum (Sg.B) evitou alteração significativa no peso corporal em todas as doses, enquanto os animais tratados com verapamil na dose de $5 \mathrm{mg} / \mathrm{kg}$ apresentaram redução significativa no peso corporal $(p<0,05)$. Os animais nos grupos tratados com verapamil (ambos, 10 mg/kg e 15 mg/kg) não apresentaram alteração significativa no peso corporal (Tabela 1).

\section{Estudos in vitro}

\section{Estudos de reatividade vascular in vitro}

Em aortas isoladas do grupo normotenso, a acetilcolina causou relaxamento completo com valor de $\mathrm{CE}_{50}$ de $0,2 \mu \mathrm{M}$ $(0,1-0,3)$ (Figura 3$)$. Por outro lado, as aortas de ratos do grupo controle hipertenso exibiram apenas 5,5\% de relaxamento dependente de acetilcolina, como mostrado na Figura 3. O tratamento com extrato bruto de $S$. guttatum de $100 \mathrm{mg} / \mathrm{kg}$ e $150 \mathrm{mg} / \mathrm{kg}$ restaurou parcialmente o relaxamento induzido por acetilcolina para 38,5 \% e 45,5\%, respectivamente. Entretanto, os anéis de ratos SD tratados com 300 mg/kg de extrato bruto de $S$. guttatum mostraram $100 \%$ de relaxamento dependente de acetilcolina, com valor de $\mathrm{CE}_{50}$ de 0,6 $\mu \mathrm{M}$ $(0,3-1,0)$ (Figura 3). O tratamento com verapamil $5 \mathrm{mg} / \mathrm{kg}$ causou apenas um relaxamento insignificante, enquanto o tratamento com $10 \mathrm{mg} / \mathrm{kg}$ induziu relaxamento de até $16 \%$. Curiosamente, o aumento da concentração de verapamil para $15 \mathrm{mg} / \mathrm{kg}$ não aumentou ainda mais o relaxamento induzido pela acetilcolina (Figura 3).

\section{Estudos in vitro da aorta de rato}

Estudos farmacológicos foram realizados na aorta de ratos normotensos para investigar o efeito anti-hipertensivo do extrato bruto $S$. guttatum. Relaxamentos induzidos pela adição cumulativa de extrato bruto em anéis aórticos pré-constritos com FE apresentaram um valor de $\mathrm{CE}_{50}$ de $0,15 \mathrm{mg} / \mathrm{mL}(0,10$ -
0,20) (Figura 4). Os anéis pré-tratados com L-NAME $(10 \mu \mathrm{M})$ mostraram relaxamento com valor de $\mathrm{CE}_{50}$ de $5,1 \mathrm{mg} / \mathrm{mL}(3,0$ 7,1 ) (Figura 4). O extrato bruto de $S$. guttatum não foi capaz de induzir relaxamento em anéis pré-tratados com atropina $(1 \mu \mathrm{M})$ e anéis desnudados de endotélio. O pré-tratamento com atropina $(1 \mu \mathrm{M})$ e a remoção do endotélio diminuíram o relaxamento induzido pelo extrato bruto em $26 \%$ e $14 \%$, respectivamente (Figura 4). O extrato bruto de $S$. guttatum também produziu vasorrelaxamento em anéis aórticos préconstritos com alta concentração de $\mathrm{K}^{+}$com valor de $\mathrm{CE}_{50}$ de $9,03 \mathrm{mg} / \mathrm{mL}$ (8,06-10,00). Em comparação, o verapamil relaxou as aortas pré-constritas com níveis altos de $\mathrm{K}^{+}$com valor de $\mathrm{CE}_{50}$ de 2,02 $\mu \mathrm{M}(1,02-3,02)$ (Figura 5).

\section{Estudo in vitro do átrio direito em ratos}

Tiras do átrio direito de ratos normotensos foram utilizadas para investigar os efeitos cronotrópicos e inotrópicos de $S$. guttatum. O extrato bruto mostrou uma diminuição dosedependente na força de contração e na frequência cardíaca com valor de $\mathrm{CE}_{50}$ de 2,99 mg/mL $(1,08-4,90)$ e 1,83 (1,02$2,64)$, respectivamente (Figura 6). Em tecidos pré-tratados com atropina, a diminuição da força de contração e da frequência cardíaca foi de $29 \%$ e 44\%, respectivamente (Figura 6).

\section{Discussão}

A S. guttatum tem sido tradicionalmente utilizada no tratamento de doenças do sangue. Ela contém uma grande quantidade de magnésio e potássio. ${ }^{11,15}$ Além disso, seu efeito antioxidante, espasmolítico e como bloqueador de entrada de $\mathrm{Ca}^{2+}$ já foi relatado. ${ }^{19,20}$

Plantas com propriedades antioxidantes, a dieta DASH (Dietary Approaches to Stop Hypertension.) rica em potássio e magnésio e bloqueadores de entrada de $\mathrm{Ca}^{2+}$ são recomendados para o manejo da hipertensão..$^{8,30,31}$ O presente estudo, utilizando o modelo de rato SD hipertenso, foi realizado para explorar o extrato bruto de $S$. guttatum como um potencial medicamento anti-hipertensivo. Diferentes doses de extrato bruto de $S$. guttatum foram administradas por via oral a ratos SD com hipertensão induzida por dieta

Tabela 1 - Efeito no peso corporal dos grupos controle normal, controle hipertenso e ratos tratados com diferentes doses do extrato bruto de Sauromatum guttatum (Sg.B) e verapamil. Os valores são expressos como média \pm DP $(n=5-7)$.

\begin{tabular}{llc}
\hline Grupos & Peso $(\mathbf{g})$ & Peso $(\mathbf{g})$ após 8 semanas \\
\hline Controle normal & $244,66 \pm 6,36$ & $267,61 \pm 3,08$ \\
\hline Grupo hipertenso & $249,28 \pm 3,25$ & $182,10 \pm 5,09^{* \star *}$ \\
\hline Tratado com Sg.B $100 \mathrm{mg} / \mathrm{kg}$ & $241,66 \pm 3,81$ & $245,01 \pm 4,66$ \\
\hline Tratado com Sg.B $150 \mathrm{mg} / \mathrm{kg}$ & $245,93 \pm 6,43$ & $250,90 \pm 3,53$ \\
\hline Tratado com Sg.B $300 \mathrm{mg} / \mathrm{kg}$ & $239,43 \pm 1,48$ & $248,63 \pm 4,52$ \\
\hline Tratado com Verapamil $5 \mathrm{mg} / \mathrm{kg}$ & $240,50 \pm 1,41$ & $214,23 \pm 3,53^{*}$ \\
\hline Tratado com Verapamil $10 \mathrm{mg} / \mathrm{kg}$ & $242,25 \pm 5,65$ & $247,68 \pm 2,96$ \\
\hline Tratado com Verapamil $15 \mathrm{mg} / \mathrm{kg}$ & $245,83 \pm 6,36$ & $254,48 \pm 3,32$ \\
\hline
\end{tabular}

$S g . B$ : Extrato bruto de Sauromatum guttatum. Os valores são expressos como média $\pm D P(n=5-7) .{ }^{*} p<0,05,{ }^{* *} p<0,01 e^{* * *} p<0,001$ vs. valores de pré-tratamento (análise ANOVA de uma via seguida de teste post-hoc de Tukey HSD) 


\section{Artigo Original}

A Normotensivo

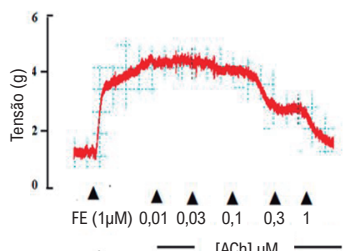

- $[\mathrm{ACh}] \mu \mathrm{M}$

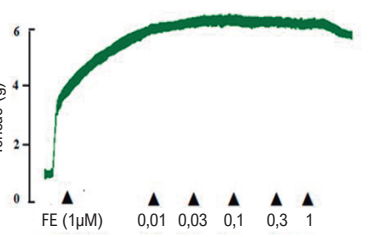

[ACh] $\mu \mathrm{M}$
B

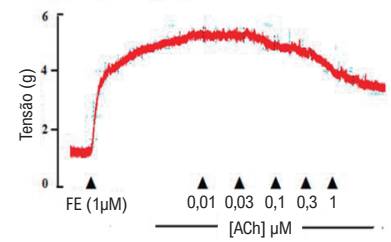

Tratado com Sg.B (150 mg/kg)

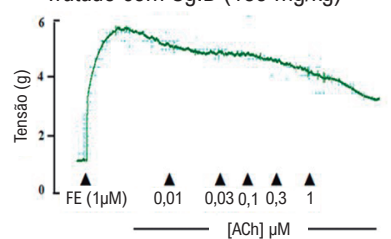

Tratado com Sg.B (300 mg/kg)

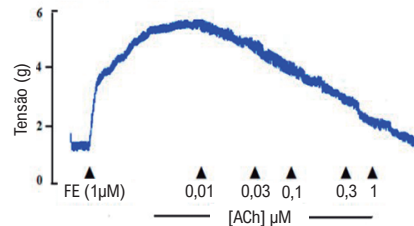

C Tratado com Verapamil $(5 \mathrm{mg} / \mathrm{kg})$

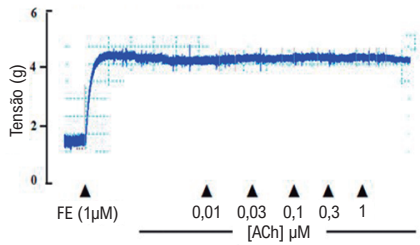

Tratado com Verapamil (10 mg/kg)

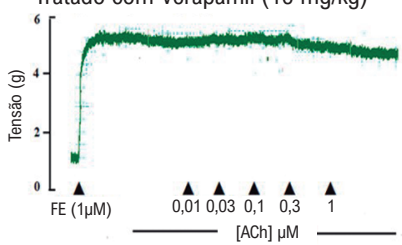

Tratado com Verapamil (15 mg/kg)

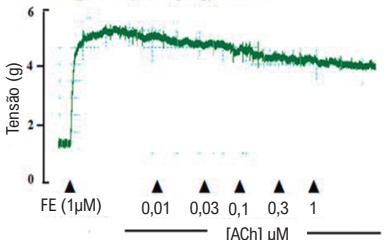

A

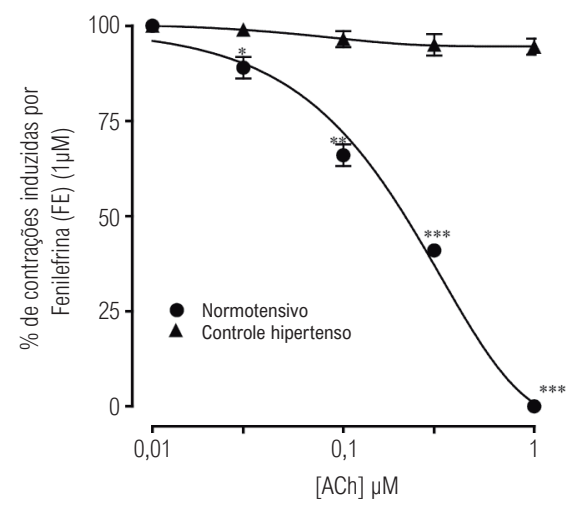

B

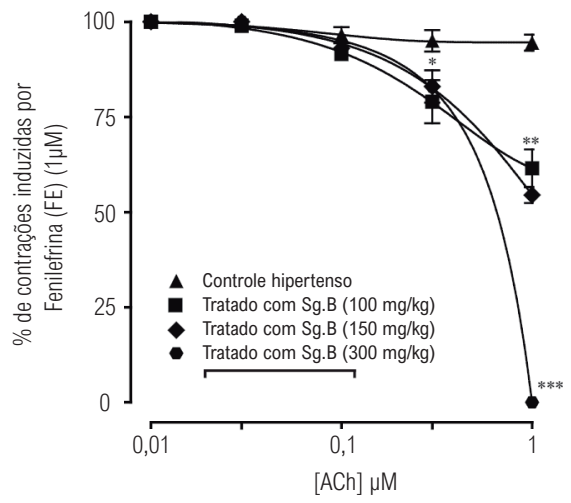

C

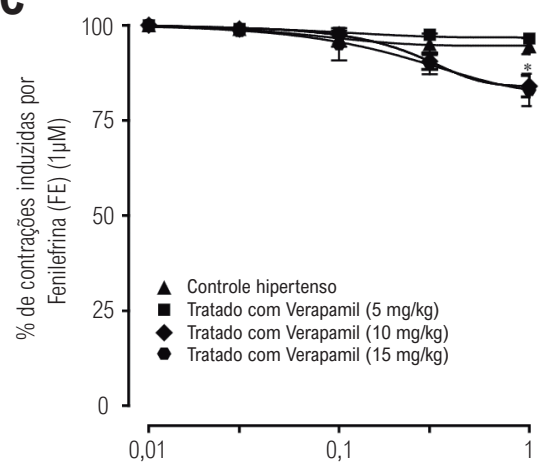

Figura 3 - Traçados e gráficos típicos mostram o efeito da acetilcolina (ACh) contra as contrações induzidas por fenilefrina nos anéis aórticos isolados de ratos normotensos, grupo controle hipertenso (A) e de ratos tratados com extrato bruto de Sauromatum guttatum (Sg.Cr) com hipertensão induzida por dieta com alto teor de sal, em doses de $100 \mathrm{mg} / \mathrm{kg}, 150 \mathrm{mg} / \mathrm{kg}$ e $300 \mathrm{mg} / \mathrm{kg}$ (B) e em ratos tratados com verapamil com hipertensão induzida por dieta com alto teor de sal em doses de $5 \mathrm{mg} / \mathrm{kg}, 10 \mathrm{mg} / \mathrm{kg}$ e $15 \mathrm{mg} / \mathrm{kg}$ (C) ( $n=5-7 ;$ média \pm DP). Em comparação com os valores do grupo controle hipertenso, ${ }^{*} p<0,05,{ }^{* *} p<0,01 e^{* * *} p<0,001$ 


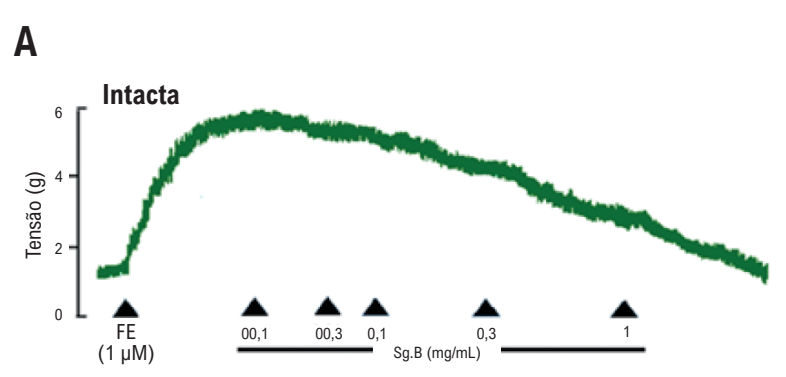

Pré-tratado com L-NAME (10 $\mu \mathrm{M})$
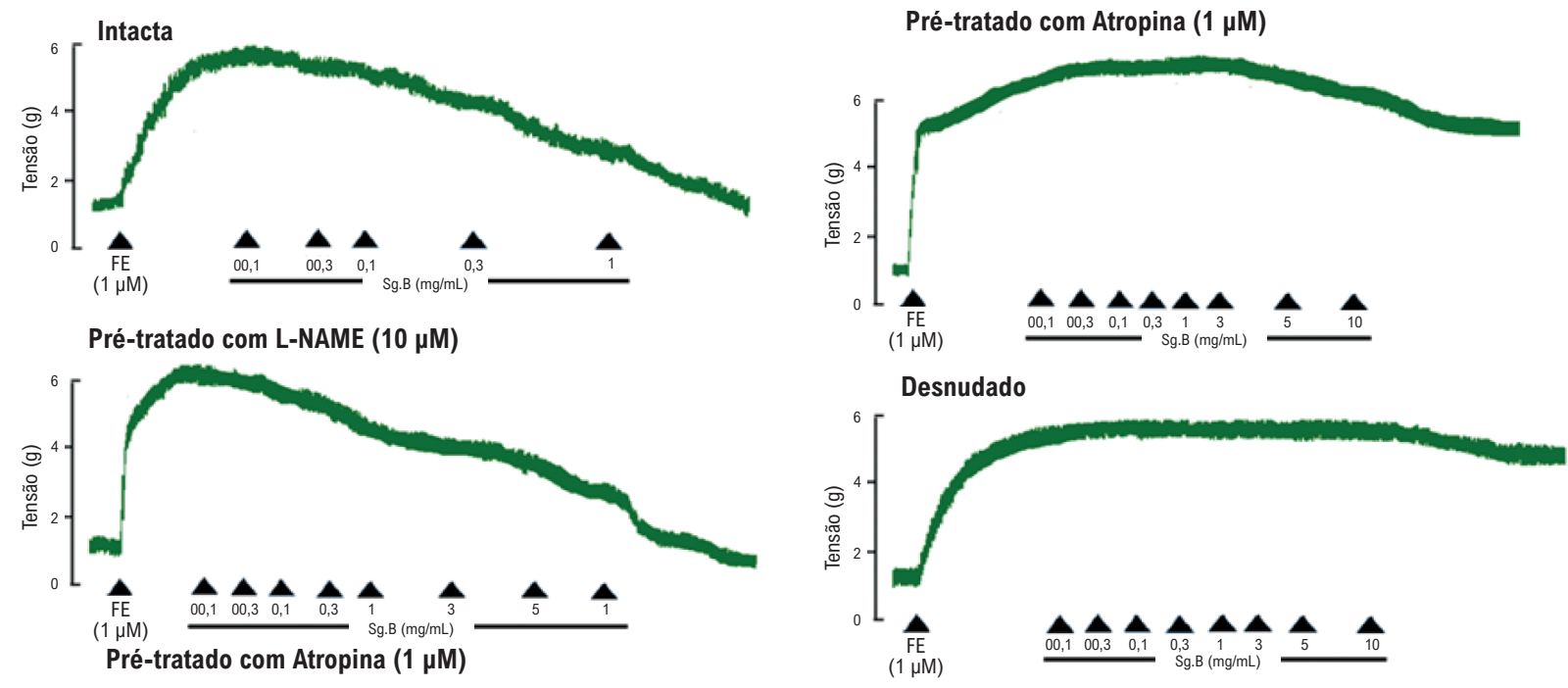

B

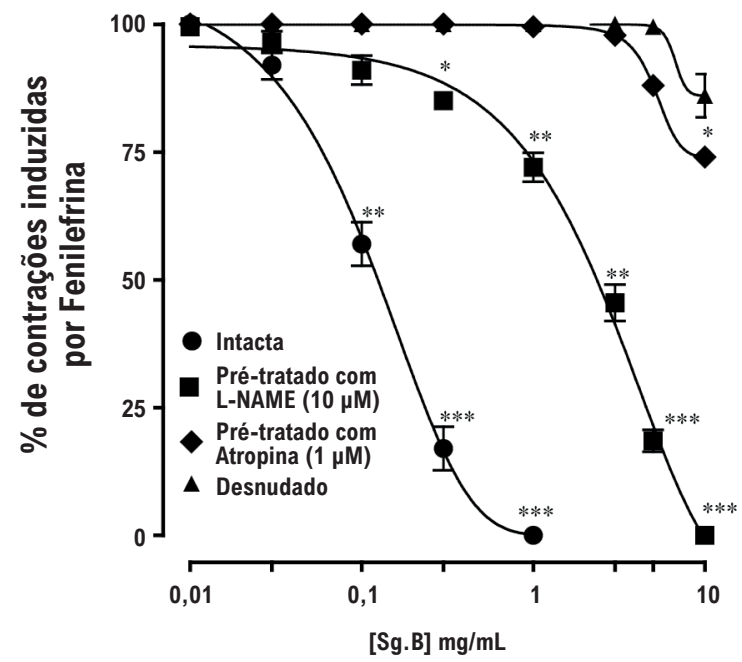

Figura 4 - 0 traçado (A) e o gráfico (B) mostram o efeito do extrato bruto de Sauromatum guttatum em aorta intacta, pré-tratada com L-NAME (10 $\mu M)$ e atropina $(1 \mu \mathrm{M})$ e em aorta de rato normotenso com endotélio desnudado versus contrações induzidas por fenilefrina $(n=5-7 ;$ média $\pm D P)$. ${ }^{*} p<0,05$, ${ }^{* *} p<0,01$ e ${ }^{* * *} p<0,001$ vs. Controle (valores pré-tratados). Análise ANOVA de duas vias seguida pelo teste post-hoc de Bonferroni.

com alto teor de sal. Esse tratamento resultou em diminuição significativa na pressão arterial média, com efeito máximo observado com uma dose de $300 \mathrm{mg} / \mathrm{kg}$. Este efeito do extrato bruto foi comparável ao do verapamil, que é um fármaco antihipertensivo padrão e bloqueador dos canais de cálcio. ${ }^{31}$ Esse achado revelou que o extrato de $S$. guttatum é eficaz contra o desenvolvimento de hipertensão experimental induzida por dieta rica em sal. No entanto, são necessários mais estudos para identificar o possível mecanismo de ação subjacente.

Uma vez que a pressão arterial é o produto de elevada resistência vascular periférica e alto débito cardíaco, ${ }^{32}$ outros experimentos foram realizados utilizando preparações vasculares e cardíacas isoladas. Primeiro, foi feita uma tentativa de estabelecer como a alta ingestão de sal induz a disfunção endotelial. A integridade do endotélio foi confirmada pela aplicação de concentrações submáximas de acetilcolina em anéis aórticos pré-constritos com fenilefrina em ratos com HIDATS. A acetilcolina não foi capaz de induzir relaxamento nos anéis aórticos do grupo de ratos com HIDATS, indicando que o endotélio foi danificado. Esse achado é corroborado por estudos anteriores. ${ }^{33-35} \mathrm{Em}$ anéis aórticos de ratos normotensos, por outro lado, as mesmas concentrações de acetilcolina induzem relaxamento, indicando a presença de endotélio funcional. Nos grupos tratados com extrato, a resposta à acetilcolina foi restaurada. Esses resultados indicam que o tratamento com extrato bruto pode reverter o dano 
A
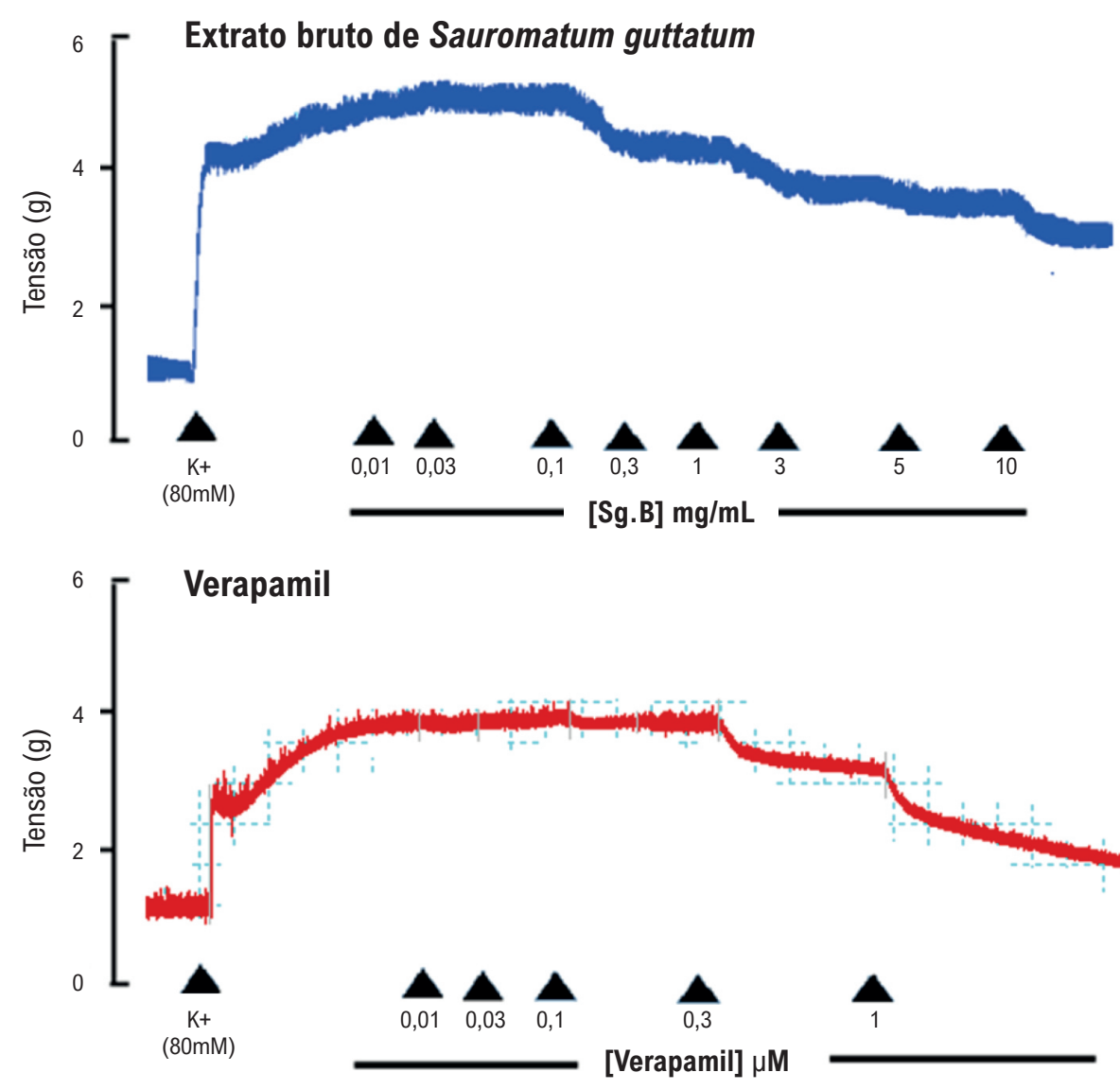

B

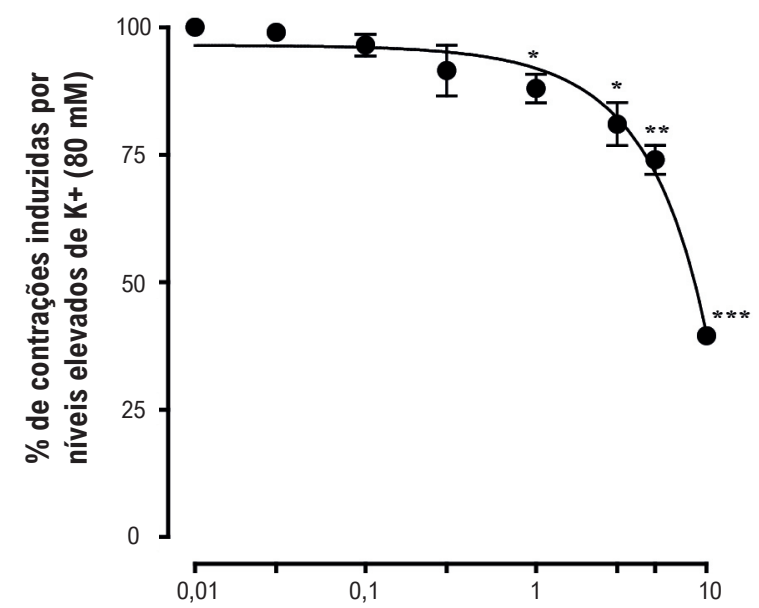

[Sg.B] $\mathrm{mg} / \mathrm{mL}$
C

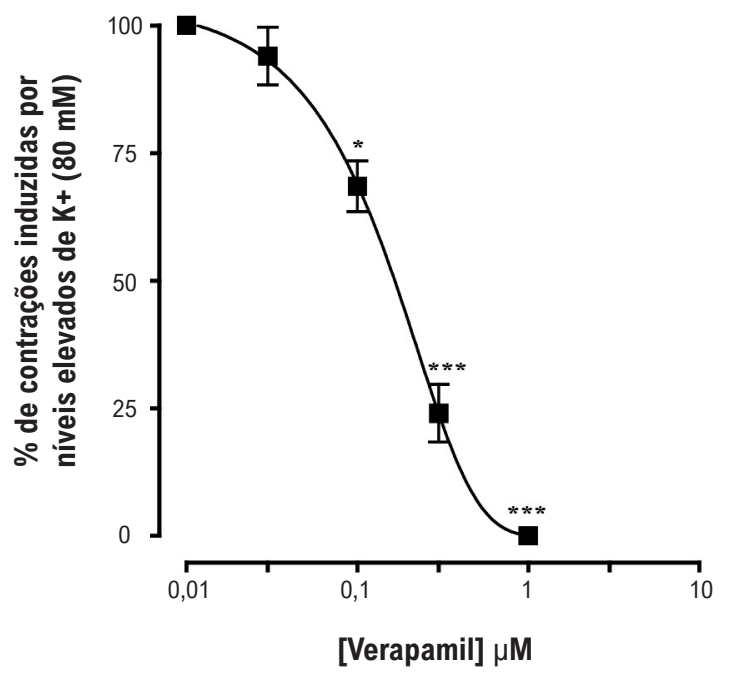

Figura 5 - 0 traçado $(A)$ e os gráficos $(B, C)$ mostram o efeito do extrato bruto de Sauromatum guttatum nas contrações induzidas por potássio elevado $\left(K^{+}\right)(80 \mathrm{mM})$ na preparação da aorta intacta de rato $(n=5-7 ;$ média $\pm D P) .{ }^{*} p<0,05,{ }^{* *} p<0,01$ e ${ }^{* \star *} p<0,001$ vs. Controle (valores pré-tratados). Análise ANOVA de duas vias seguida pelo teste post-hoc de Bonferroni. 
A
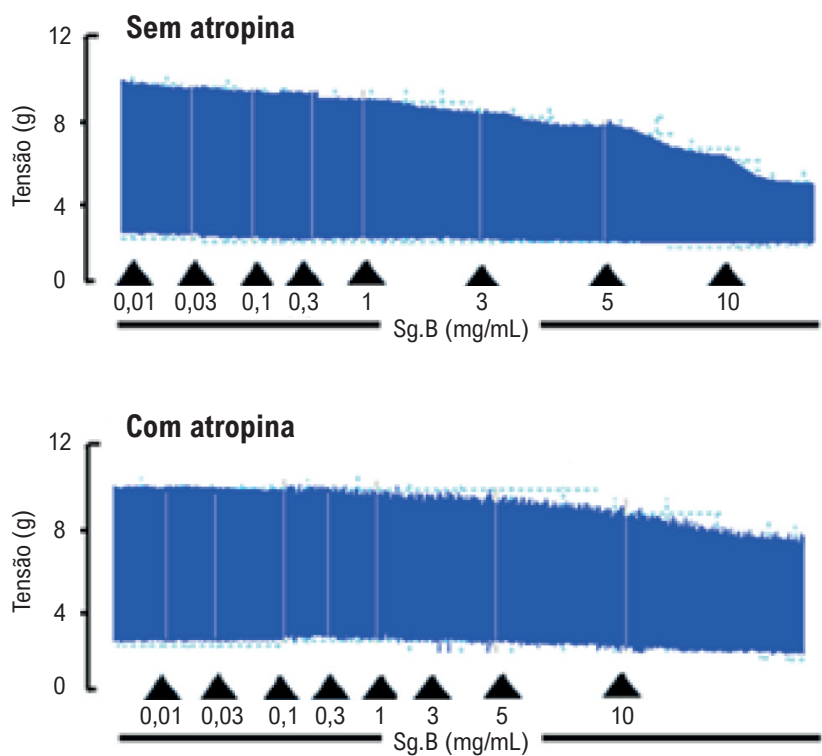

B

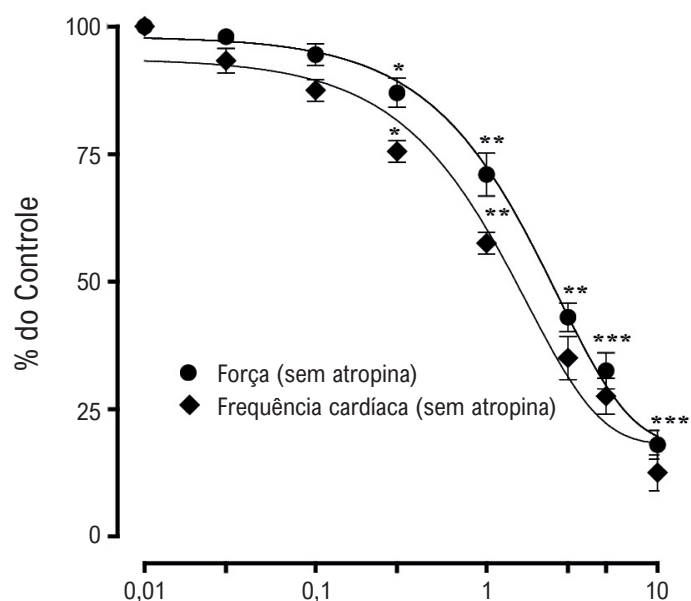

$[S g . B] \mathrm{mg} / \mathrm{mL}$

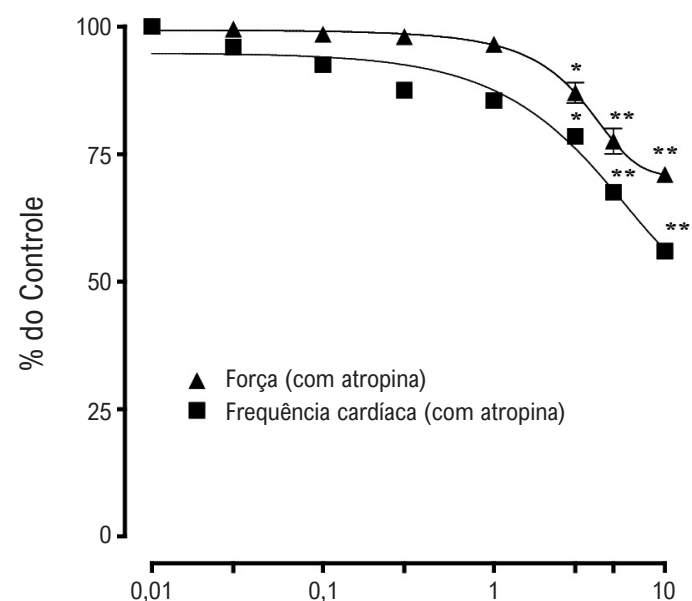

$[\mathrm{Sg} \cdot \mathrm{B}] \mathrm{mg} / \mathrm{mL}$

Figura 6 - 0 traçado $(A)$ e os gráficos $(B, C)$ mostram os efeitos inotrópico e cronotrópico do extrato bruto de Sauromatum guttatum sem e com atropina $(1 \mu M)$ no átrio direito de ratos normotensos pré-tratados ( $n=5-7$; média $\pm D P){ }^{*} p<0,05,{ }^{* *} p<0,01$ e ${ }^{* *} p<0,001$ vs. controle (valores pré-tratados). Análise ANOVA de duas vias seguida pelo teste post-hoc de Bonferroni

endotelial e também evitar a elevação da pressão arterial média observada in vivo. Em comparação, o verapamil falhou em induzir vasorrelaxamento em anéis aórticos de ratos com HIDATS do grupo controle ou ratos tratados, indicando que seu mecanismo de ação é diferente do extrato bruto. $\mathrm{O}$ extrato de $\mathrm{S}$. guttatum exerce sua função anti-hipertensiva na hipertensão experimental através da preservação parcial da função endotelial.
Outros estudos in vitro foram realizados na aorta para investigar o(s) mecanismo(s) de ação(ões) subjacente(s). Em anéis aórticos pré-constritos com acetilcolina de ratos normotensos, a adição cumulativa de concentrações de extrato bruto induziu vasorrelaxamento. A remoção do endotélio reverteu completamente esse efeito, sugerindo que fatores derivados do endotélio vascular podem desempenhar um 
papel. No entanto, a alta concentração de acetilcolina ainda induziu relaxamento, sugerindo envolvimento de diferentes mecanismos. Para estudar o envolvimento do óxido nítrico, os anéis aórticos foram pré-tratados com L-NAME, um inibidor de óxido nítrico sintase. ${ }^{36}$ Curiosamente, o efeito vasorrelaxante do extrato de $S$. guttatum foi reduzido em cerca de $75 \%$ na concentração de $1 \mathrm{mg} / \mathrm{mL}$, embora em concentrações mais altas ele tenha desviado a curva de resposta para a direita. Esses achados sugerem que o extrato de $S$. guttatum induz vasorrelaxamento através tanto da via dependente do endotélio (em concentração mais baixa) como da via independente do endotélio (em concentração mais alta). O componente dependente do endotélio pode ser atribuído ao óxido nítrico. Em células endoteliais vasculares, a liberação de óxido nítrico é acoplada a receptores muscarínicos. ${ }^{37}$ Para verificar se o efeito do extrato bruto de S. guttatum está ligado a receptores muscarínicos e ao óxido nítrico, os anéis aórticos foram contraídos com atropina, um antagonista do receptor muscarínico. ${ }^{37}$ Este pré-tratamento aboliu o vasorrelaxamento associado ao extrato bruto de $S$. guttatum, indicando assim uma ação através da via do óxido nítrico (NO) ligada ao receptor muscarínico. A Atropina ou o L-NAME não foram capazes de inibir o relaxamento em concentrações mais elevadas do extrato bruto, sugerindo ainda que o extrato também pode atuar nos músculos lisos vasculares. Para testar essa hipótese, anéis aórticos foram contraídos com alta concentração de $\mathrm{K}^{+}$. Curiosamente, a adição cumulativa do extrato bruto induziu um efeito vasorrelaxante 10 vezes menos potente do que contra a FE. O K+ elevado foi utilizado para induzir contrações, pois ativa os canais de cálcio dependentes de voltagem (Cav) e a liberação de $\mathrm{Ca}^{2+}$ através da despolarização, resultando em vasoconstrição. ${ }^{38,39}$ Esses achados indicam que o extrato bruto de $\mathrm{S}$. guttatum também inibe a entrada de $\mathrm{Ca}^{2+}$ pelos canais dependentes da voltagem. Também sugere que o $\mathrm{NO}$ vascular desempenha um papel dominante no efeito vasorrelaxante e anti-hipertensivo de $S$. guttatum, além do efeito nos músculos lisos vasculares.

Para investigar o efeito do extrato de $S$. guttatum nos parâmetros cardíacos, foram utilizadas tiras atriais isoladas de ratos. O extrato de $S$. guttatum mostrou efeitos negativos inotrópicos (82\%) e cronotrópicos (56\%) quando adicionado cumulativamente às tiras do átrio direito que se contraem espontaneamente. Para testar o possível papel dos receptores muscarínicos cardíacos, as tiras atriais foram pré-tratadas com atropina. Este pré-tratamento inibiu parcialmente o efeito do extrato bruto de $S$. guttatum, indicando assim a possibilidade de que o efeito negativo inotrópico ou cronotrópico observado seja devido à ativação de receptores muscarínicos cardíacos. Entretanto, nossos achados revelaram que o extrato é mais seletivo para os receptores muscarínicos vasculares do que para os receptores cardíacos.

O extrato de $S$. guttatum também foi testado quanto à presença de constituintes fitoquímicos. Foi verificado que ele contém flavonoides, fenóis e taninos. Estudos anteriores revelaram o efeito terapêutico de flavonoides, fenóis e taninos na hipertensão. ${ }^{40-42}$ Portanto, esses constituintes podem ser os agentes ativos responsáveis pela redução da pressão arterial e efeitos vasculares na hipertensão induzida por alto teor de sal. Os estudos fitoquímicos futuros serão concentrados no isolamento dos componentes ativos e na exploração dos mecanismos subjacentes, como o bloqueio do cálcio e a via do óxido nítrico a nível molecular.

\section{Conclusão}

Esses achados indicam que $S$. guttatum possui atividade anti-hipertensiva, resultando em efeitos vasodilatadores e depressores do miocárdio atrial ligados a receptores muscarínicos. A preservação da função endotelial, a liberação de NO dependente do receptor muscarínico e a inibição do movimento de $\mathrm{Ca}^{+2}$ são os mecanismos subjacentes de vasodilatação. O extrato de $S$. guttatum também exerce efeitos negativos inotrópico e cronotrópico, possivelmente devido à ativação de receptores muscarínicos cardíacos. Nossos resultados, observados no modelo de rato SD, fornecem uma explicação farmacológica para o potencial anti-hipertensivo de $S$. guttatum.

\section{Agradecimentos}

O manuscrito foi revisado para o inglês por Tamas Kriska, PhD, Departamento de Farmacologia e Toxicologia, Medical College of Wisconsin, Watertown Plank Road 8701, WI, EUA. Os autores gostariam de agradecer a Tamas Kriska, PhD (tkriska@mcw.edu) por sua assistência.

\section{Contribuição dos autores}

Concepção e desenho da pesquisa e Obtenção de financiamento: Bibi R, Shah AJ; Obtenção de dados, Análise e interpretação dos dados e Redação do manuscrito: Bibi R, Salma U, Bashir K, Khan T, Shah AJ; Análise estatística: Bibi R, Salma U, Shah AJ; Revisão crítica do manuscrito quanto ao conteúdo intelectual importante: Bibi R, Salma U, Shah AJ.

\section{Potencial conflito de interesse}

Não há conflito com o presente artigo

\section{Fontes de financiamento}

O presente estudo não teve fontes de financiamento externas.

\section{Vinculação acadêmica}

Este artigo é parte de dissertação de mestrado de Bibi Rabia pelo COMSATS Institute of Information Technology Abbottabad Campus.

\section{Aprovação ética e consentimento informado}

Este estudo foi aprovado pelo Comitê de Ética do COMSATS University Islamabad, Abbottabad Campus, Pakistan sob o número de protocol EC/PHM/07-2013/CIIT/ ATD. Todos os procedimentos envolvidos nesse estudo estão de acordo com a Declaração de Helsinki de 1975, atualizada em 2013. 


\section{Referências}

1. Chobanian AV, Bakris GL, Black HR, Cushman WC, Green LA, Izzo Jr JL, Jones DW, Materson BJ, Oparil S, Wright Jr JT, Roccella EJ. The seventh report of the joint national committee on prevention, detection, evaluation, and treatment of high blood pressure: the JNC 7 report. Jama. 2003; 289(19):2560-71.

2. Freis ED. The role of salt in hypertension. Blood pressure. 1992; 1(4):196-200.

3. Dahl LK. Possible role of salt intake in the development of essential hypertension. Int J Epidemiol. 2005; 34(5):967-72

4. Feng W, Dell'Italia LJ, Sanders PW. Novel paradigms of salt and hypertension. Journal of the American Society of Nephrology. 2017; 28(5):1362-9.

5. Carvalho MV, Siqueira LB, Sousa AL, Jardim PC. The influence of hypertension on quality of life.Arq Bras Cardiol. 2013; 100(2):164-74.

6. Pagan LU, Gomes MJ, Okoshi MP. Endothelial function and physical exercise. Arq Bras Cardiol.. 2018; 111(4): 540-1.

7. Gilani AH, Atta-ur-Rahman. Trends in ethnopharmacology. Ethnopharmacol. 2005; 100(1-2): 43-9.

8. Carey RM, Whelton PK. Prevention, detection, evaluation, and management of high blood pressure in adults: synopsis of the 2017 American College of Cardiology/American Heart Association hypertension guideline. Ann Intern Med. 2018; 168(5): 351-8.

9. Dass S, Mathur M. Herbal drugs: Ethnomedicine to Modern Medicine. Ramawat KG. ISBN: 9783540791164. New York, USA: Springer; 2009.

10. Patale C, Nasare P, Narkhede S. Ethnobotanical studies on the medicinal plants of Darekasa Hill range of Gondia district, Maharashtra, India. Int J Res Plant Sci. 2015; 5: 10-6.

11. Quattrocchi U. CRC World Dictionary of Medicinal and Poisonous Plants. Philadelphia: CRC Press;2016.ISBN: 9780429171482.

12. Smith BN, Meeuse BJ. Production of volatile amines and skatole at anthesis in some arum lily species. Plant Physiol. 1966; 41(2): 343-7.

13. Chen J, Meeuse B. Production of free indole by some aroids. Acta Botanica Neerlandica. $1971 ; 20(6)$ : 627-35.

14. Borg-Karlson AK, Englund FO, Unelius CR. Dimethyl oligosulphides, major volatiles released from Sauromatum guttatum and Phallus impudicus. Phytochemistry. 1994; 35(2): 321-3.

15. Khan T, Ahmad M, Khan H, Ahmad W. Standardization of crude extracts derived from selected medicinal plants of Pakistan for elemental composition using SEM-EDX. Asian J Plant Sci. 2006; 5: 211-6.

16. SinghBains J, Singh J, Kamboj SS, Nijjar KK, Agrewala JN, Kumar V, et al. Mitogenic and anti-proliferative activity of a lectin from the tubers of Voodoo lily (Sauromatum venosum). Biochim Biophys Acta (BBA) 2005; 1723(1-3):163-74.

17. Khan T, Ahmad M, Khan R, Khan H, Choudhary MI. Phytotoxic and insecticidal activities of medicinal plants of Pakistan: Studies on Trichodesma indicum, Aconitum laeve and Sauromatum gutatum. J Chem Soc Pakistan. 2007; 29(3): 260-4.

18. Khan T, Ahmad M, Ahmad W, Saqib QNU, Choudhary MI. Preliminary evaluation of the antispasmodic and lipoxygenase inhibitory effects of some selected medicinal plants. Pharmaceut Biol. 2009; 47(12): 1137-41.

19. Abbasi MA, Shahwar D, Wahab M, Saddiqui MF. Antibacterial and antioxidant activities of an ethnobotanically important plantSauromatum venosum (Ait.) Schott. of District Kotli, Azad Jammu \& Kashmir. Pakistan J Botan. 2011; 43(1): 579-85.

20. Shah N, Shah AJ, Ahmed M, Gilani AH. Functional nature of the spasmolytic effect, phytochemical composition and acute toxicity studies on Sauromatum guttatum. Bangl J Pharmacol. 2014; 9(2): 203-7.

21. Kaur M, Thakur K, Kamboj SS, Kaur S, Kaur A, Singh J. Assessment of Sauromatum guttatum lectin toxicity against Bactrocera cucurbitae. J Environ Biol. 2015; 36(6); 1263.
22. Williamson EM, Okpako DT, Evans FJ. Pharmacological Methods in Phytotherapy Research. New York: John Wiley \& Sons Ltd; 1996.

23. Edeoga HO, Okwu DE, Mbaebie BO. Phytochemical constituents of some Nigerian medicinal plants. African Journal of Biotechnology. 2005; 4(7): 685-688

24. National Research Council. Guide for the Care and Use of Laboratory Animals Washington DC, National Academy Press; 2011.

25. Salma U, Khan T, Shah AJ. Antihypertensive effect of the methanolic extract from Eruca sativa Mill., (Brassicaceae) in rats: Muscarinic receptor-linked vasorelaxant and cardiotonic effects. J Ethnopharmacol. 2018; 224: 409-20.

26. Lawler JE, Sanders BJ, Chen YF, Nagahama S, Oparil S. Hypertension produced by a high sodium diet in the borderline hypertensive rat (BHR). Clin Experimental Hypertens. Part A: Theory and Practice. 1987; 9(11):1713-31.

27. Vasdev S, Gill V, Longerich L, Parai S, Gadag V. Salt-induced hypertension in WKY rats: prevention by a-lipoic acid supplementation. Mol Cel Biochem. $2003 ; 254(1-2): 319-26$

28. Qayyum R, Qamar HMUD, Khan S, Salma U, Khan T, Shah AJ. Mechanism underlying the antihypertensive properties of Urtica dioica. Journal of Translational Medicine. 2016; 14(1): 254.

29. Furchgott RF, Zawadaski JV. The obligatory role of endothelial cells in the relaxation of arterial smooth muscle by acetylcholine. Nature. 1980 299: 373-6.

30. Baradaran A, Nasri H, Rafieian-Kopaei M. Oxidative stress and hypertension: Possibility of hypertension therapy with antioxidants. J Res Med Sci. 2014; 19(4): 358

31. Godfraind T. Discovery and development of calcium channel blockers. Front in Pharmacol. 2017; 8: 286

32. VincentJL. Understanding cardiac output. Crit Care. 2008; 12(4): 174

33. Luscher TF, Vanhoutte PM, Raij L. Antihypertensive treatment normalizes decreased endothelium-dependent relaxations in rats with salt-induced hypertension. Hypertension. 1987; 9(6):III193.

34. Miyoshi A, Suzuki H, Fujiwara M, Masai M, Iwasaki T. Impairment of endothelial function in salt-sensitive hypertension in humans. Am J Hypertens. 1997; 10(10):1083-90.

35. Banday AA, Muhammad AB, FaziliFR, Lokhandwala M. Mechanisms of oxidative stress-induced increase in salt sensitivity and development of hypertension in Sprague-Dawley rats. Hypertension. 2007; 49(3):664-71.

36. KüngCF,Moreau P, Takase H, Lüscher TF. L-NAME hypertension alters endothelial and smooth muscle function in rat aorta: prevention by trandolapril and verapamil. Hypertension. 1995; 26(5): 744-51.

37. Hammer R, Giachetti A. Muscarinic receptor subtypes: $M_{1}$ and $M_{2}$ biochemical and functional characterization. Life Scienc. 1982; 31(26): 2991-8.

38. Nishimura K, Ota M, Ito K. Existence of two components in the tonic contraction of rat aorta mediated by $\alpha_{1}$ adrenoceptor activation. $\mathrm{Br}$ Pharmacol. 1991; 102(1): 215-21.

39. Godfraind T. EDRF and cyclic GMP control gating of receptor-operated calcium channels in vascular smooth muscle. Eur J Pharmacol. 1986; 126(3): 341-3.

40. Bhargava UC, Westfall BA. The mechanism of blood pressure depression by ellagic acid. Proceeding of the Society for Experimental Biology and Medicine. 1969; 132(2): 754-6.

41. MolineJ, Bukharovich IF, WolffMS, Phillips R. Dietary flavonoids and hypertension: is there a link? Med Hypotheses. 2000; 55(4): 306-9.

42. Godos J, Sinatra D, Blanco I, Mulè S, La Verde M, Marranzano M. Association between dietary phenolic acids and hypertension in a Mediterranean cohort. Nutrients. 2017; 9(10): 1069. 